\title{
OFICINA DE APOIO AOS ESTUDOS (OFAPE): PROCEDIMENTOS DE AUTORREGULAÇAO PARA O ENGENHEIRO DO AMANHÃ
}

\author{
Miguel Angel Chincaro Bernuy - migueltrabalho@gmail.com \\ Rosângela Borges Pimenta - rosangelaborges.pimenta@gmail.com \\ Luciana Carneiro Hernandes - luciana@utfpr.edu.br \\ Lucas Rosalin Chaves de Souza - lucaschavesdesousa@gmail.com \\ Jhonatan Felipe Pereira-jhowpereira2@gmail.com \\ Pedro Paulo Noguerol Odorizzi-pedro_odorizzi@hotmail.com \\ Universidade Tecnológica Federal do Paraná \\ Avenida Alberto Carazzai, 1640, Centro \\ 86300000 - Cornélio Procópio - Paraná
}

Resumo: Considerando que o engenheiro do amanhã necessita construir competências que lhe permitam um contínuo desenvolvimento técnico-científico e humanístico, para solucionar situações de complexidade intrínseca à sociedade digital, é relevante que sejam organizadas estratégias de formação destes engenheiros com esta finalidade. Se por um lado, as reorganizações curriculares trabalham para organizar o contexto desse desenvolvimento, são necessárias ações de microplanejamento no contexto das rotinas do dia a dia desses estudantes. Por outro lado, o desempenho dos estudantes precisa ser observado da mesma forma, para promover a excelência e a autonomia dos egressos. Nesta perspectiva, este trabalho apresenta um conjunto de procedimentos na forma de oficinas, que foram aplicados por estudantes para estudantes e analisados para o desenvolvimento da autorregulação, tendo como princípio o desenvolvimento da capacidade de organizar os estudos. Entre as análises obtidas tem-se a percepção crescente da obtenção da capacidade de planejamento para os estudos após a realização das oficinas, tanto nos estudantes que ofertaram quanto para os que participaram.

Palavras-chave: Autorregulação. Planejamento de Estudos. Aprendizagem.

\section{INTRODUÇÃO}

Nas últimas décadas, fatores como fracasso escolar, repetência e evasão estão presentes na maioria dos contextos escolares, causando grande preocupação aos educadores (SIMÃO et al, 2015). O baixo rendimento acadêmico é resultante de inúmeros fatores que atingem os estudantes universitários. Um deles é a forma de organizar e se preparar para os estudos. A grande maioria não consegue obter um bom resultado nas avaliações por não saber como se preparar. Segundo o MEC (1996), um dos fatores característicos individuais dos estudantes de graduação, referente à evasão, está relacionado às habilidades de estudo. A constatação desta insuficiência aponta a necessidade de um maior preparo, para que não ocorram as dependências nas disciplinas e, consequentemente, uma possível desistência do curso. O desempenho acadêmico exerce um papel fundamental na relação do aluno com a universidade e com a escolha de sua carreira. Quando insatisfatório, pode levar o estudante à desmotivação, bem como gerar insegurança com relação às suas competências. (BARDAGI E HUTZ,2012). Com base neste cenário, o aporte teórico desta pesquisa está centrado nos construtos da autorregulação e das técnicas de estudo.

A autorregulação relaciona-se à competência dos estudantes quanto à proatividade durante a aprendizagem e ao controle de "seus processos cognitivos, metacognitivos e motivacionais", 
a fim de "adquirirem, organizarem e transformarem as informações adquiridas ao longo do tempo" (FREIRE, 2009, p.276). A aprendizagem autorregulada inclui três fases diferentes: 1) estabelecimento e planejamento de metas antes de estudar; 2) uso de estratégias diferentes; monitoramento e controle da aprendizagem como um estudo; e 3) reflexão sobre a aprendizagem após o estudo (ZIMMERMAN e SCHUNK, 2012).

Muitos estudos apontam a autorregulação da aprendizagem como conceito fundamental no processo de construção de conhecimento por considerar "o indivíduo como um sujeito que atua e sofre influências de diferentes dimensões, como ambientais, pessoais e comportamentais" (BASSO; ABRAHÃO, 2017). Ao proporcionar uma aprendizagem mais autônoma e ativa, em que o aluno consegue mobilizar os conhecimentos apreendidos e criar estratégias para enfrentar as diversas situações a ele apresentadas, a autorregulação é considerada como força motriz para o aprendizado profundo e longevo, "ao longo de toda a vida" (COSNEFROY, 2013 apud BASSO; ABRAHÃO, 2017).

Esse processo, bastante dinâmico, permite que o estudante gerencie "os sistemas de planejamento, organização, controle e avaliação dos processos adotados, os resultados atingidos e as variáveis contextuais" (SILVA; VEIGA SIMÃO, 2016 apud BASSO; ABRAHÃO, 2017) e envolve a interação de múltiplas variáveis como conhecimento, competência e motivação, aspectos bastante relacionados ao processo de autorreflexão.

Inerentes à autorregulação da aprendizagem, a definição dos objetivos e o gerenciamento do tempo são considerados por muitos autores como imprescindíveis para que o estudo se dê de maneira mais autônoma e proativa (COSNEFROY, 2013; FRISON; VEIGA SIMÃO, 2011 apud BASSO; ABRAHÃO, 2017). Também integram o processo autorregulatório a seleção de estratégias adequadas para a execução dos objetivos, a revisão sistemática dos mesmos e os redirecionamentos necessários, ou seja, "existe um caráter fundamentalmente voluntário e intencional subjacente a esse conceito" (FREIRE, 2009, p.280).

Uma pesquisa sobre competências de estudo, realizada com 126 universitários da área de ciências exatas, identificou que os estudantes autorregulam seus estudos utilizando mais estratégias voltadas a ações, tais como: anotações em aula, leituras adicionais e exercícios, e menos em relação a estratégias metacognitivas voltadas a aprender como aprender.(JOLY et al, 2015). Esta problemática ocupa lugar de destaque na reforma do ensino superior, pois é fundamental que os estudantes tenham êxito na universidade, com foco na busca de estratégias educativas que os auxiliem nos estudos e com o seu desenvolvimento na transição para a vida adulta (MAGALHÃES,2012).

Paralelamente à autorregulação, temos a prática como "o maior aliado para o aperfeiçoamento da aprendizagem acadêmica" e os projetos de extensão como fator bastante relevante para "aprimorar o conhecimento adquirido ao longo da graduação, além de melhorar a autoconfiança, proporcionar o conhecimento profissional na área escolhida, melhorando o currículo e aumentando as chances para o mercado de trabalho" (DESLANDES; ARANTES, 2017, p.181).

Assim, tendo em vista a necessidade do "ensino de estratégias que permitam enfrentar os imprevistos, o inesperado e a incerteza, modificando seu desenvolvimento, em virtude das informações adquiridas ao longo do tempo" (MORIN, 2001 apud FREIRE, 2009, p.277) para que a autorregulação da aprendizagem se efetive, discentes da graduação da UTFPR-CP propuseram a realização de oficinas que propiciassem o desenvolvimento das competências autorregulatórias em si mesmos e em outros discentes.

O objetivo do presente artigo é relatar a experiência da realização de Oficinas de Apoio aos Estudos (OFAPE), desenvolvidas junto a graduandos da Universidade Tecnológica Federal do Paraná, Câmpus Cornélio Procópio. Tal proposta se inseriu no âmbito do Grupo de Estudos 
sobre Metodologias Aplicadas às Engenharias, mediante aprovação do Edital Protagonismo Estudantil. A primeira etapa deste projeto, que teve início em 2019, consistiu em uma pesquisa sobre os fatores críticos do rendimento acadêmico na formação universitária. As técnicas de estudo serão aqui apresentadas, como forma de autorregulação, juntamente com discussões e considerações acerca dessa experiência de intervenção no contexto acadêmico.

\section{TÉCNICAS DE ESTUDO}

Esta seção tem por propósito apresentar a fundamentação da pesquisa feita para a escolha das metodologias que seriam abordadas nas oficinas. Deste modo, buscaram-se no campo do conhecimento as principais referências na definição de conceitos, bem como a aplicação das metodologias de aprendizagem.

No decorrer das oficinas foram utilizados dez métodos, alguns dos quais validados historicamente (Resumos, Listas de exercícios, Teste prático e Anotações em sala) e outros mais contemporâneos, descritos abaixo.

\subsection{Mapa mental}

O mapa mental foi desenvolvido na década de 1970 pelo psicólogo inglês Tony Buzan (BUZAN, 2005), que queria aperfeiçoar as etapas de aprendizagem e memorização. Seu estudo levou a um método que tentava simular a forma com que o cérebro humano trabalha e armazena informações nos neurônios, como uma árvore que tem um caule principal e, a partir dele, várias ramificações - o princípio dos mapas mentais, nos quais uma ideia central decompõe-se em ramificações e interligações. Os mapas mentais hierarquizam as informações, tornando-as mais fáceis para a sua identificação e classificação. "É uma técnica de registro visual e conceitual de informações" (HERMANN e BOVO, 2006 p.91).

\section{Passos para a utilização da técnica}

Para a criação de um Mapa Mental (MM), Tony Buzan desenvolveu sete passos para a sua elaboração. Sintetizando as etapas de Buzan temos que: os Mapas Mentais devem começar no centro de uma folha de papel em branco na horizontal, recomenda-se utilizar imagens ou figuras sempre que puder como ideia central acompanhadas de cores em todo o processo. Após colocado o assunto principal do MM precisa-se ligar os ramos principais, secundários e terciários sempre obedecendo uma ordem de prioridade. Buzan ressalta que todas os ramos devem ser curvos e não em linha reta tendo em vista que ramos curvos são mais atraentes e estimulante para o cérebro e para termos uma eficácia sempre utilizar palavras-chaves que serão desencadeadoras de associações e conexões.

\subsection{Pomodoro}

A técnica Pomodoro foi criada na década de 1980 pelo italiano Francesco Cirillo (CIRILLO, 2006). Criada com o intuito de aumentar a produtividade dos estudos a partir da gestão do tempo, quando Francesco estava no primeiro ano da universidade. A priori, para a execução da técnica, foi utilizado um timer de cozinha em forma de tomate - pomodoro, em italiano - instrumento prosaico que nomeou o método. 


\section{Passos para a utilização da técnica}

A técnica Pomodoro consiste em um método para aprimorar a gestão de tempo, em que dividimos o ato do estudo em intervalos de 25 minutos focando completamente em ler o conteúdo, realizar atividades e afins e outro intervalo de 5 minutos para descanso. Dessa forma, o estudante começa a realizar seu estudo de uma maneira menos cansativa e consegue gerar um maior foco na hora de estudar.

\subsection{EPL2R}

A técnica EPL2R, conhecida em inglês por SQ3R ou também como método Robinson (BECK, 2020), foi criada na década de 1946 pelo psicólogo educacional Francis Pleasante Robinson, com o intuito de se desenvolver um maior aproveitamento no momento de estudos. Para tanto, Robinson propõe que se divida o ato de estudar em 5 etapas.

\section{Passos para a utilização da técnica}

O método de estudo pela técnica EPL2R consiste em dividir o estudo em cinco etapas. O primeiro é da "Exploração": neste momento o estudante deve ler o seu material de forma superficial, para que, desta forma, possa estar realizando a segunda etapa que é "Perguntar", neste momento a partir da primeira leitura o praticante deve formular perguntas com relação ao conteúdo lido, assim na terceira etapa que é uma leitura ativa, o estudante deverá sanar as perguntas e questionamentos que foram criados. A quarta etapa, "Rememorar" consiste em escrever o conteúdo aprendido, gerando assim uma memória de longo prazo, podendo utilizar por exemplo mapas mentais e resumos do conteúdo. A última etapa, "Repassar", consiste em transmitir o conhecimento adquirido para outras pessoas.

\subsection{Aula invertida}

A metodologia Aula Invertida foi criada entre os anos de 2007 e 2008, na Wooddland Park High School, Colorado, Estados Unidos, pelos professores de química Jonatham Bergmann e Aaron Sams (FERRARINI, 2019, p.19). O intuito foi que os alunos pudessem aprender em seu próprio ritmo. Vale ressaltar, que a metodologia foi adaptada pelo grupo para ser utilizada voluntariamente, sem o auxílio de um professor.

\section{Passos para a utilização da técnica}

A sala de aula invertida é uma nova forma de promover a aprendizagem, em que o aluno é ativo neste processo. O estudante estuda antes da aula e a aula se torna um lugar de aprendizagem ativa, onde há perguntas, discussões e atividades práticas.

Os estudantes levarão para os momentos de aula presencial suas impressões, dúvidas e experiências, ganhando assim, um tempo com o professor para auxiliá-lo. Além disso, o aluno tem condição de estudar de acordo com seu ritmo de produtividade com essa técnica, pois, ele sempre estará adiantado no conteúdo.

Para obter resultados de aprendizagem mais significativos é importante realizar a combinação de várias atividades: leitura, demonstração, discussão, prática e ensino da matéria aprendida para outras pessoas. Contudo, o fato de já ter estudado a matéria, antes dela ter sido dada na aula presencial, despertará um interesse maior nas aulas presenciais para tirar dúvidas, então o tempo na sala de aula também será bem utilizado.

Sendo assim, atividades ativas, a exemplo da sala de aula invertida, têm o poder de tornar o ensino um processo completo e mais motivador em que o aluno observa uma razão para a 
aplicação dos conhecimentos e, com isso, consegue reter uma quantidade muito mais significativa de informação e aumentar seu desempenho nos estudos.

\subsection{Teste prático}

O estudo de Lilienfeld (LILIENFED, 2000) descobriu que o Teste Prático é duas vezes mais eficiente que qualquer outra técnica de estudo. Pode-se afirmar que é uma das melhores técnicas de aprendizagem.

\section{Passos para a utilização da técnica}

Os psicólogos responsáveis pela pesquisa apontam que a qualidade dessa técnica se deve aos vários formatos possíveis de aplicação: questões de múltipla escolha, testes do tipo "preencha a lacuna", questões assertivas. Ao final de cada conteúdo, são feitos os testes práticos para exercitar aquilo que acabou de estudar. Quanto mais testes e exercícios forem resolvidos mais eficácia terá a técnica.

\section{PROCESSO DE ORGANIZAÇÃO DAS OFICINAS}

O contexto de partida para a criação das oficinas foi a percepção que o rendimento dos estudantes estava comprometido, observando os indicadores de retenção e evasão na UTFPRCP. Segundo dados do Departamento de Educação, para um curso a retenção total de estudantes cresceu até $20,6 \%$ e a retenção parcial cresceu até 54,8\% (DEPED-CP, 2019).

$\mathrm{Na}$ perspectiva de amenizar essa situação, o Grupo de Estudos sobre Metodologias Aplicadas às Engenharias começou a pesquisar técnicas que, otimizando o aprendizado do alunado via organização de tempo e modo, poderiam incentivar os discentes a seguir construindo conhecimentos na universidade.

Assim, foram estudados artigos científicos e analisadas diversas técnicas de estudo, entre outros materiais indicados pelos orientadores. Considerando a efetividade e a facilidade de execução de cada técnica, o grupo selecionou as que poderiam ser mais bem recebidas pelos estudantes, tais como: EPL2R, Pomodoro, Teste Prático, Aula Invertida, Mapas Mentais, Anotações em Aula, Resumos, Listas de Exercícios, Estudo Diário e Otimização de Tempo.

Decidido a ofertar aos discentes uma possibilidade de escolha entre as várias técnicas que agregariam a esperada produtividade nos estudos, o grupo buscou a melhor maneira de apresentá-las, elaborando o material para as oficinas, planejando o formato do curso, escolhendo o que seria utilizado a cada dia.

Sistematizado o material - com conceito das técnicas, formulários, textos para aplicação, rodas de conversa, exposição de ideias e dinâmicas - foram realizados testes, nos quais os estudantes ministrantes apresentaram as aulas elaboradas para os orientadores, a fim de realizar os ajustes necessários depois do feedback recebido.

Por fim, com o cronograma pronto, prevendo os encontros semanais, teve início a divulgação das oficinas para os alunos da universidade, por e-mail e a realização das inscrições via Google Forms. As oficinas contemplaram, ao todo, sete semanas, sendo um dia de abertura e um dia de encerramento; cinco dias foram utilizados para apresentação dessas técnicas, incluindo também uma aula de organização e planejamento. Cada oficina teve a duração de 1 hora. 


\subsection{Construção das atividades das oficinas}

A rotina de desenvolvimento das atividades das oficinas foi realizada de duas maneiras. Inicialmente, por meio de reuniões presenciais e, em seguida, por meio de reuniões remotas mediante programas de comunicação síncrona (Skype e Meet).

Durante as reuniões, além das revisões de conteúdo e formatação, foram observados dois aspectos que permitiram a construção do conhecimento de forma prática, ou seja, o primeiro aspecto a acessibilidade dos conceitos envolvidos e suas etapas correspondentes, e o segundo aspecto a aplicação das técnicas.

\section{REALIZAÇÃO DAS OFICINAS}

A Oficina de Apoio aos Estudos (OFAPE) está lançando sua terceira edição. Em um primeiro momento, a mesma foi desenvolvida de forma presencial, com alunos em sala; e, a partir da segunda, realizada remotamente, de forma totalmente virtual devido à nova situação ocasionada pelo isolamento social decorrente da pandemia da COVID19.

Em síntese, no diagrama da Figura 1 estão representadas as principais realizações de cada dia.

Figura 1 - Representação de cada dia da Oficina com suas respectivas ações.

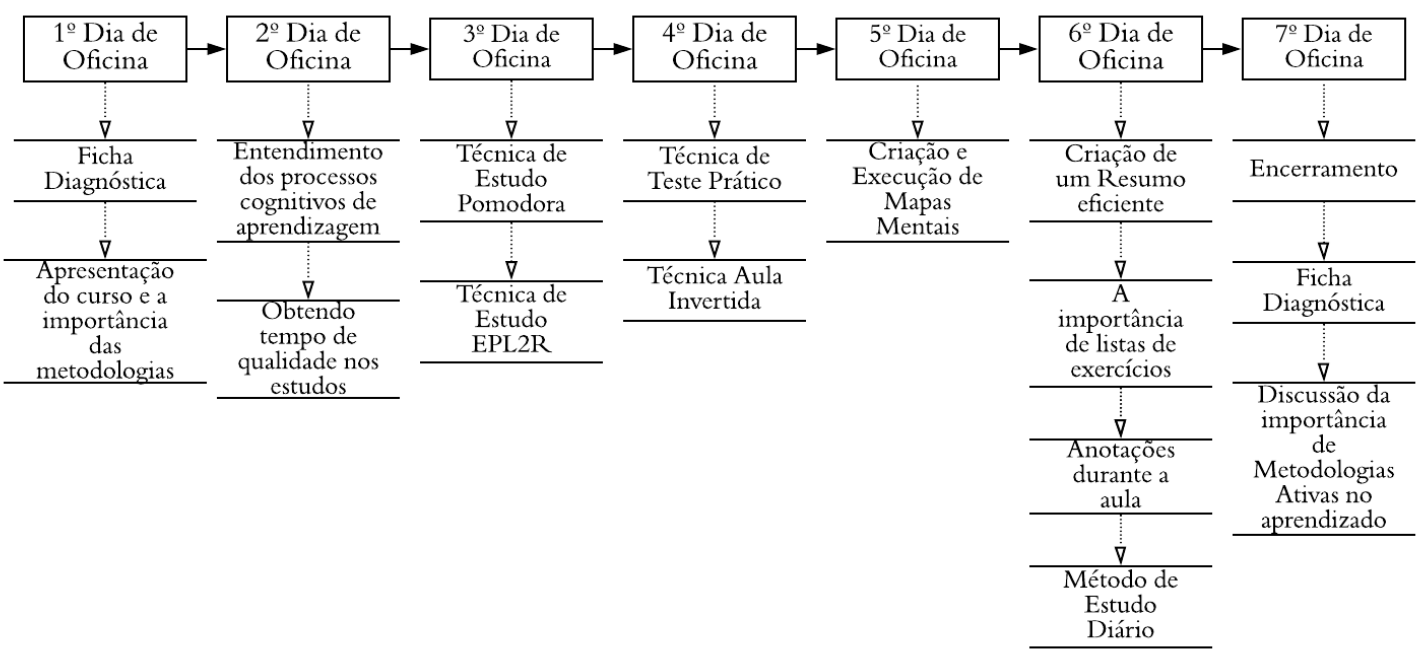

Fonte: Autores, 2020.

No primeiro dia, os participantes das Oficinas preencheram uma ficha diagnóstica para coleta de dados no que tange às Técnicas de Estudo e ao conhecimento que os alunos tinham sobre esse assunto. Também foram introduzidos conceitos sobre formas de estudo não mais centradas somente no professor e em aulas expositivas.

$\mathrm{Na}$ outra semana, foi discutida a maneira pela qual os estudantes aprenderiam de forma mais efetiva e como assimilariam conteúdos nesta nova era digital, pois é de extrema importância que os alunos entendam como se dá todo o processo de aprendizado e assimilação de conteúdo pelo funcionamento do cérebro humano. Em seguida, foram apresentados possíveis meios para otimizar e construir um tempo realmente de qualidade para o estudo.

Nas demais semanas foram efetivamente apresentadas várias técnicas de estudo e suas respectivas formas de aplicação. Logo depois da explanação das técnicas, os alunos foram encorajados a colocarem cada uma em prática para perceberem a melhora gradativa no 
rendimento acadêmico. Vale ressaltar que toda a oficina foi moldada para atender ao novo cenário em que os alunos e professores estão inseridos.

No último encontro foi entregue a mesma ficha diagnóstica do primeiro dia para que os alunos relessem os próprios relatos e, depois do contato com todas as técnicas e com a aplicação das mesmas, refletissem sobre o rendimento acadêmico e o conhecimento construído nos dois meses de contato com a OFAPE, sendo possível a eficácia da oficina e das metodologias ativas de aprendizagem para as Engenharias.

\section{ANÁLISE DOS DADOS}

O Gráfico 1 mostra as porcentagens de alguns dados coletados pelo questionário antes e depois de serem aplicadas as técnicas de estudo, para a otimização do tempo e o entendimento do processo de aprendizagem. Mediante estes dados, fica evidente que se obteve uma melhora significativa no rendimento acadêmico somente participando das oficinas e aplicando as técnicas de estudo apresentadas, visto que 32,3\% dos participantes alegaram que não tinham uma rotina de estudo, $44,5 \%$ relataram que não possuíam nenhuma forma de planejamento para a vida acadêmica e $34 \%$ declaram que a sua forma de estudo não atendia as suas necessidades acadêmicas. E logo depois das oficinas serem ministradas, esses dados cairam de maneira satisfatória para $24,3 \%, 3 \%$ e 5,8\%, respectivamente, consolidando o objetivo da criação da oficina que, por meio de recursos de aprendizagem, pode-se obter uma ascensão na produtividade acadêmica.

Outro dado interessante para destaque é a disseminação e aplicação das metodologias de ensino, como é mostrado no Gráfico 1 . Somente 32,3\% já conheciam e utilizavam as mesmas e este número cresceu para 50,3\% mostrando novamente uma eficácia da técnica.

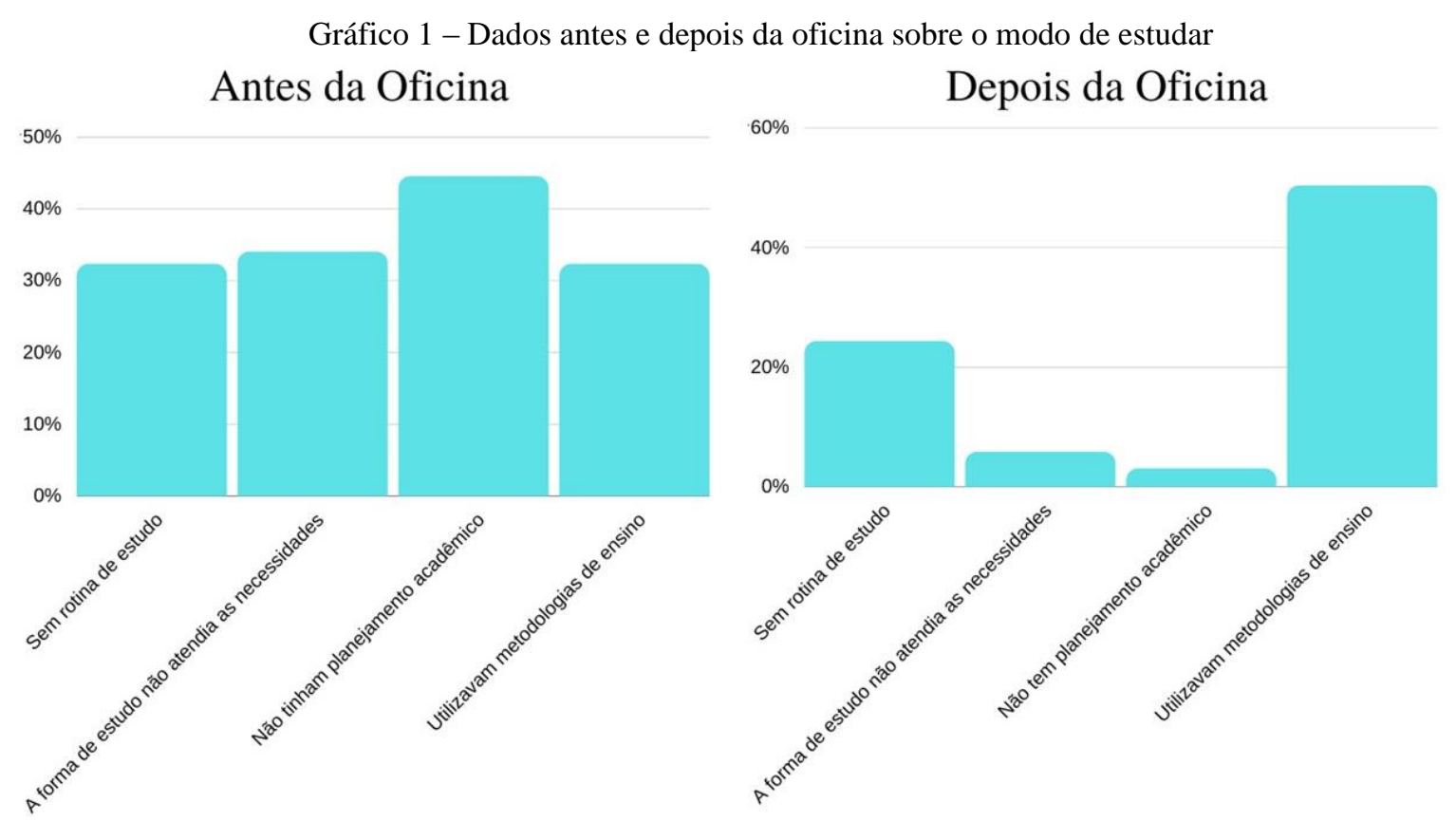

Fonte: Autores, 2020

Por intermédio do Gráfico 2, percebe-se que a oficina teve impacto na forma de estudo dos participantes, pois $63,9 \%$ afirmam que ocorreram mudanças em sua forma de aprender e $100 \%$ 
passaram a considerar um plano de estudo como algo relevante na hora de estudar, dentre os quais $69,4 \%$ consideraram muito relevante a aplicação de um esquema de aprendizagem.

Gráfico 2 - Representação de cada dia da Oficina com suas respectivas ações.

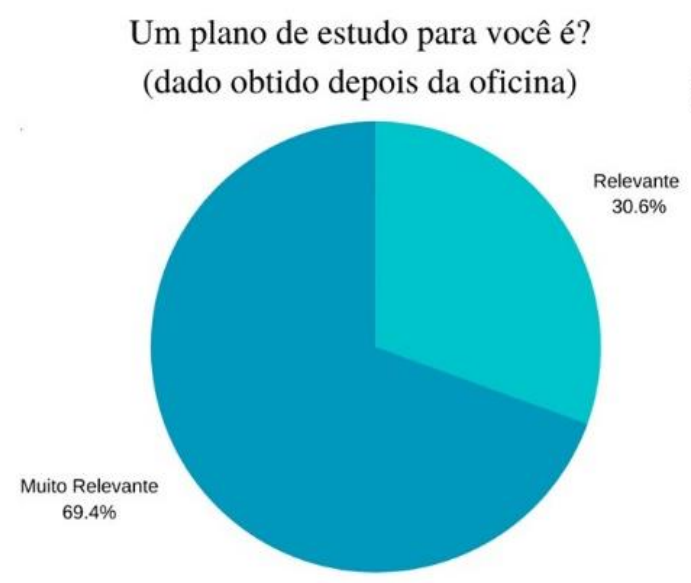

Houve mudanças na sua forma de estudo?

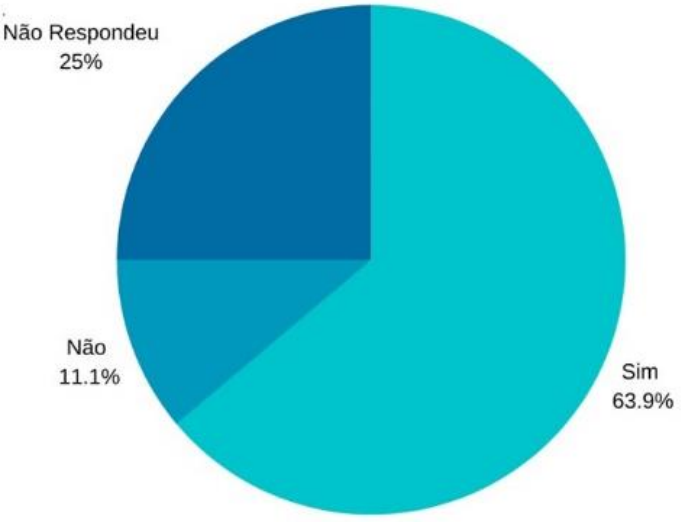

Fonte: Autores, 2020

\section{CONSIDERAÇÕES FINAIS}

Pode-se considerar que os objetivos esperados foram atingidos, pois a maioria dos alunos, mais de $60 \%$, afirmam que ao término da oficina ocorreram mudanças em sua forma de estudar. Portanto, é notório o fato de que as oficinas impactaram positivamente a rotina de estudo dos participantes.

Espera-se que, com futuras aplicações da oficina, um número maior de alunos seja atingido e que, com aplicação das técnicas para otimização dos estudos sendo aplicada em larga escala, os índices de evasão e retenção sejam minimizados.

A realização das oficinas trouxe benefícios não apenas para os alunos participantes, mas também para os responsáveis pelo seu desenvolvimento. Melhorias na oratória, no desenvolvimento de habilidades de comunicação, na destreza para a confecção de materiais para as aulas, dentre outras, são algumas das capacidades e habilidades desenvolvidas pelos estudantes que ministraram as aulas. Portanto, o aprendizado neste caso ocorreu como uma via de mão dupla, em que todos os envolvidos receberam algo para alavancar suas carreiras.

\section{Agradecimentos}

Agradecemos a Universidade Tecnológica Federal do Paraná pela infraestrutura disponibilizada para o desenvolvimento de diversas atividades apresentadas neste trabalho, por meio de um Projeto de Ensino Homologado em Edital da DIRGRAD-CP.

\section{REFERÊNCIAS}

BARDAGI, M. P.e HUTZ, C. S. Mercado de trabalho, desempenho acadêmico e o impacto sobre a satisfação universitária. Revista de Ciências Humanas, 46(1), 183-198. doi:10.5007/2178-4582.2012v46n1, 2012.

BASSO, Fabiane P.; ABRAHÃO, Maria Helena M. B. Autorregulação da aprendizagem em contexto escolar: uma abordagem baseada em Ateliês Biográficos de Projetos. Educar em 
Revista, Curitiba, Brasil, Edição Especial n. 1, p. 171-189, jun. 2017. Disponível em: https://www.scielo.br/scielo.php?pid=S010440602017000500171\&script=sci_abstract\&tlng= pt. Acesso em: 27 jul. 2020.

BECK, Caio. Método de Leitura SQ3R. Andragogia Brasil, 2018 Disponível em: https://andragogiabrasil.com.br/metodo-de-leitura-sq3r/. Acesso em 27 jul. 2020.

BUZAN, Tony. Mapas Mentais e sua Elaboração: um sistema definitivo de pensamento que transformará a sua vida. São Paulo: Cultrix, 2005. Disponível em: https://books.google.com.br/books?hl=ptBR\&lr=\&id=lBC4bNHiWYgC\&oi=fnd\&pg=PA40

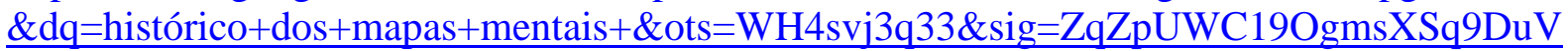
Kn0ryrQ\#v=onepage\&q\&f=false. Acesso em: 23 jul. 2020.

CIRILlO, Francesco. The Pomodoro Techinique. San Francisco Califórnia: Creative Commons, 2006. Disponível em: https://lasolutionestenvous.com/wpcontent/uploads/2014/04/ThePomodoroTechnique_v1-3.pdf. Acesso em: 27 jul. 2020.

DEPED-CP, Seminários de Projetos Pedagógicos - NDE Engenharia Elétrica da UTFPR, Cornélio Procópio, $2019 . \quad$ Disponível em: https://drive.google.com/file/d/1xsbk1eWif2f0jXvJeT-CgZ_-OIuJTs52/view?usp=sharing. Acesso em: 27 jul. 2020.

DESLANDES, Maria S. S.; ARANTES, Álisson R. A extensão universitária como meio de transformação social e profissional. Sinapse Múltipla, 6(2), dez., 179-183, 2017. Disponível em: http://periodicos.pucminas.br/index.php/sinapsemultipla. Acesso em: 27 jul. 2020.

DULOSKY, John et all. Improving Students' Learning With Effective Learning Techniques: Promising Directions From Cognitive and Educational Psychology. Sage Journal, volume 14, 08 jan. 2013, p 4-58. Disponível em: //journals.sagepub.com/doi/ https 10.1177/1529100612453266. Acesso em: 27 jul. 2020.

FERRARINI, Rosilei; SAHEB, Daniele; TORRES, Patricia Lupion. Metodologias ativas e tecnologias digitais: aproximações e distinções. Revista Educação em Questão, Natal, v. 57, n. 52, p. 1-30, e- 15762, abr./jun. 2019.

FREIRE, Luiz Gustavo Lima. Auto-regulação da aprendizagem. Ciência \& Cognição, Rio de Janeiro , v. 14, n. 2, p. 276-286, jul. 2009. Disponível em: http://pepsic.bvsalud.org/scielo.php?script=sci_arttext\&pid=S1806-58212009000200019. Acesso em: 20 jul. 2020.

JOLY, Maria Cristina Rodrigues Azevedo et al. Competência de estudo para uma amostra universitária da área de exatas. Psicologia Escolar e Educacional, Maringá v.19, n.1, 23-29, Jan./Apr.,2015.

HERMANN,W.; BOVO,V. Mapas mentais: enriquecendo inteligências: captação,seleção,organização,síntese, criação e gerenciamento de informação.2 ed.Campinas:Walther Hermann\& Viviani Bovo, 2005. 
LILIENFELD, Scott O.; WOOD, James M.; GARB, Howard N. The scientific status of projective techniques. Psychological science in the public interest, v. 1, n. 2, p. 27-66, 2000.

MAGAlHÃES, Cleidilene Ramos. A autorregulação da aprendizagem em Programa Institucional de Acolhimento e Suporte ao aluno universitário: os professores como parceiros. Pelotas, vol 42 : 143 - 167, maio/junho/julho/agosto, 2012.

MEC. Diplomação, retenção e evasão nos cursos de graduação em instituições de ensino superior públicas. Avaliação: Revista de rede de avaliação institucional da educação superior. Campinas, v. 1, n. 2, p. 55-65, dez. 1996.

SIMÃO, Ana Margarida da Veiga.; FRISON, Lourdes Maria Bragagnolo.; NONTICURI, Amélia Rodrigues. Dos significados à autorregulação: perspectivas de estudantes com trajetórias acadêmicas de insucesso. Revista de Estudios e Investigatión em Psicología Y Educación,v.n 1,2015.

ZIMMERMANN, Barry, J.; SCHUNK, Dale, H. Motivation: An essential dimension of selfregulated learning. (Eds.).Motivation and self-regulated learning. Theory, research and applications, Routledge, pp.1-30, New York, 2012.

\title{
STUDY SUPPORT WORKSHOP (OFAPE): SELF-REGULATION PROCEDURES FOR FORECOMING ENGINEER
}

\begin{abstract}
Once considered that the forecoming engineer needs to build up abilities which allow him/her a lifelong humanistic as well as scientific-technical development to provide solutions to situations whose complexity is intrinsic to the digital society, it is relevant to set strategies of development regarding the formation of such individuals with such objective. If, on the one hand, the curricular remaking works in the sense of providing the context for such development, on the other, careful thorough action is necessary in the scope of the daily routine of such students. In such perspective, this work presents a range of procedures by means of workshopscarried on by students for students and analyzed for the accomplishment of self-regulationwhich have been founded in the best principle of compiling the practice of studies. Among the collected data one may base the statement that it is perceivable the increasingly higher ability to plan for studying after the workshops both in the students which applied the method as well as in those who took part as proper object of that practice.
\end{abstract}

Keywords: Self-regulation. Study Planning. Learning. 